\title{
Approaches to Multireceptor Targeting: Hybrid Radioligands, Radioligand Cocktails, and Sequential Radioligand Applications
}

\author{
Jean Claude Reubi ${ }^{1}$ and Helmut R. Maecke ${ }^{2}$ \\ ${ }^{1}$ Institute of Pathology, University of Berne, Berne, Switzerland; and ${ }^{2}$ Department of Nuclear Medicine, University Hospital Freiburg, \\ Freiburg, Germany
}

\begin{abstract}
Modern drug discovery highly depends on the identification and validation of the drug targets. Using the method of in vitro quantitative receptor autoradiography, we demonstrated that-for instance, in neuroendocrine tumors - up to 3 receptors can be coexpressed at a relatively high density. In addition, nonendocrine tumors such as breast, prostate, and brain tumors concomitantly express several G protein-coupled receptors at a high density. We propose 3 strategies for exploiting these findings for multireceptor targeting in vivo: use of heterobivalent or heteromultivalent ligands, which may bind simultaneously or monovalently to their different molecular targets; coinjection of a cocktail of radioligands; and sequential injection of different radioligands. Any of these strategies may help to remedy some of the major problems in cancer targeting: heterogeneity, change in phenotype during disease progression, and resistance.
\end{abstract}

Key Words: neuroendocrine tumors; quantitative autoradiography; heterobivalent ligands; cocktail approach; tumor heterogeneity

J Nucl Med 2017; 58:10S-16S

DOI: 10.2967/jnumed.116.186882

W

hen a cancer target expresses a sufficient amount of receptors, it is possible to target it with radiolabeled compounds having a high affinity for those receptors. In the peptide receptor field - the focus of this review-the past 2 decades have shown that the somatostatin receptors expressed in neuroendocrine tumors (NETs) can be successfully targeted with various somatostatin analogs (1).

Until recently, the clinical targeting evaluation was limited to a specific receptor (i.e., somatostatin receptor $2\left[\mathrm{sst}_{2}\right]$ ) expressed in a cancer type and therefore was limited to the use of a single radioligand for a given cancer. However, here we discuss the possibility of using multireceptor targeting for well-defined cancer types. We present preclinical data demonstrating multiple peptide receptor expression in specific human tumors, measured in vitro using binding methods such as autoradiography, a method shown in the last 2 decades to be predictive for in vivo applications. We first summarize the receptor data for NETs and then expand the discussion to non-NETs, which represent a much larger cancer

Received Jun. 7, 2017; revision accepted Jul. 19, 2017.

For correspondence or reprints contact: Helmut R. Maecke, Department of Nuclear Medicine, University Hospital Freiburg, Hugstetter Strasse 55, 79106 Freiburg, Germany.

E-mail: helmut.maecke@uniklinik-freiburg.de

COPYRIGHT (C) 2017 by the Society of Nuclear Medicine and Molecular Imaging. population. We focus mainly on peptide receptors, a group of targets particularly well investigated in vitro in cancer using receptor binding (2). We also introduce the observation that antagonists may allow the identification of more receptors in a target than agonists do, therefore increasing the scope of target indications (3). We use these data as a basis to discuss possible approaches for multireceptor targeting in vivo in clinics. We also discuss the main advantages of heterobivalent or heteromultivalent ligands, which may bind simultaneously to different receptors, as well as the cocktail approach and a modification of that approach (sequential application of different imaging agents).

\section{RECEPTOR EXPRESSION IN CANCER}

\section{NETs}

Gastroenteropancreatic (GEP) NETs. Thirty years ago, specific peptide receptors-namely, somatostatin receptors-were identified as targets in cancers (2). This identification led to the development of radiolabeled somatostatin analogs suitable for binding to overexpressed receptors and for imaging tumors. Later on, it was shown that not only $\mathrm{sst}_{2}$ but also several other somatostatin receptor subtypes ( $\mathrm{sst}_{3}$ and $\mathrm{sst}_{5}$ ) could be expressed concomitantly in a single NET tumor sample (4), introducing the idea that multireceptor targeting within the somatostatin receptor family may be feasible. A compound with an affinity for $\mathrm{sst}_{2}, \mathrm{sst}_{3}$, and $\mathrm{sst}_{5}$ DOTANOC-was developed and shown to label $\mathrm{sst}_{2^{-}}, \mathrm{sst}_{3^{-}}$, and sst $_{5}$-positive tumors (5), often detecting in vivo more sites than $\mathrm{sst}_{2}$-selective ligands (6).

Recent in vitro studies have shown that, apart from somatostatin receptors, other peptide receptors are overexpressed in NETs. In particular, the incretin receptor glucagonlike peptide 1 (GLP-1) receptor is massively overexpressed in insulinomas $(7,8)$. The clinical development of ${ }^{111}$ In-DOTA-exendin- 4 and ${ }^{68} \mathrm{Ga}$-exendin-4 targeting the GLP-1 receptor has been highly successful in insulinomas (9). Within the incretin family, another receptor was recently shown to be overexpressed in most gastrointestinal and pancreatic NETs: the glucose-dependent insulinotropic polypeptide (GIP) receptor (10). Preclinical evidence has shown that ${ }^{68} \mathrm{Ga}$-DOTA-GIP can label GIP receptor-positive cancers in animals, although this method is not yet used in clinics (11). In addition, cholecystokinin (CCK) receptors (CCK1 and CCK2 subtypes) are overexpressed in GEP NETs $(7,12)$, with CCK2 being overexpressed more frequently than CCK1. CCK2 receptors were imaged in GEP NET patients (13). Finally, we recently showed in vitro (Jean Claude Reubi and Beatrice Waser, unpublished data, 2017) that the glucagon receptor is also overexpressed in GEP NETs, in particular, in insulinomas.

Of particular interest is the observation that the aforementioned peptide receptors can be concomitantly overexpressed in many 
GEP NETs (Table 1; Figs. 1A, 1B, and 1C). Moreover, a recent autoradiography study measuring GLP-1 receptors, GIP receptors, and $\mathrm{sst}_{2}$ concomitantly in the same GEP NET sections showed that the 3 types of receptors were often coexpressed (14). In particular, it was found that the use of the 3 respective radioligands allowed acquisition of a highly homogeneous tumor labeling in cases in which the use of a single radioligand would show only a heterogeneous patchy labeling. Of equal importance, the use of the 3 radioligands concomitantly increased the total labeling of a tumor. The triple labeling also permitted identification of all of the tested tumors as positive cases, whereas the use of a single radioligand revealed several negative cases in this series (14). Clinical testing using either a cocktail of the aforementioned 3 radioligands or a monomer triagonist radioligand has not yet been documented.

Medullary Thyroid Carcinomas (MTC). MTC is another NET that may be multitargeted by radiolabeled peptides. It is well known that more than $90 \%$ of the MTC overexpress the CCK2 receptor (12). Proof of concept has been established in vivo in patients (13) with various CCK analogs, however, a marked kidney uptake has made a routine application difficult. Recently, it has been observed that receptors for the incretin GIP were also overexpressed in $89 \%$ of MTC (15). Figure 1D shows an MTC that expresses both CCK2 and GIP receptors.

\section{Non-NETs}

An increasing amount of in vitro studies have shown in the past 2 decades that numerous non-NETs can overexpress peptide (and other) receptors. In many cancer types, 2 or more peptide receptor types can be concomitantly expressed: the present review will focus on prostate cancer, breast cancer, gastrointestinal stromal tumors (GISTs), Ewing sarcomas, and glioblastomas.

\section{Prostate Cancer}

Prostate cancer expresses gastrin-releasing peptide (GRP) receptors in more than $90 \%$ of the primary tumor (16), including tumors at a very early stage of development (17). Metastases, in particular hormone-independent metastases, appear to express GRP receptors-less often than primary tumors, however (16). Of particular interest is the high expression of the prostate-specific membrane antigen (PSMA), which is also overexpressed in most prostate cancers (18). Clinical investigations have shown that both

TABLE 1

Selection of Peptide Receptors Coexpressed in Specific Cancer Types

\begin{tabular}{|c|c|c|c|}
\hline Cancer type & Peptide receptor & Incidence in cancer & References \\
\hline \multicolumn{4}{|l|}{ NETs } \\
\hline \multirow[t]{5}{*}{ Ileal } & Somatostatin $\left(\mathrm{sst}_{2}>\mathrm{sst}_{3}>\mathrm{sst}_{5}\right.$ or $\left.\mathrm{sst}_{1}\right)$ & $\mathrm{sst}_{2}>90 \%$ & 4,7 \\
\hline & CCK2 & $60 \%$ & 7,12 \\
\hline & GIP & $86 \%$ & 10,14 \\
\hline & GLP-1 & $30 \%$ & $7,8,14$ \\
\hline & Glucagon & $35 \%$ & \\
\hline \multirow[t]{4}{*}{ Insulinomas } & GLP-1 & $>90 \%$ & $7,8,14$ \\
\hline & GIP & $100 \%$ & 10,14 \\
\hline & Glucagon & $>90 \%$ & \\
\hline & Somatostatin ( $\left.\mathrm{sst}_{1}, \mathrm{sst}_{5}, \mathrm{sst}_{2}\right)$ & $70 \%$ & 4,7 \\
\hline \multirow[t]{3}{*}{ MTC } & CCK2 & $>90 \%$ & 12 \\
\hline & GIP & $89 \%$ & 15 \\
\hline & Somatostatin $\left(\mathrm{sst}_{2}\right)$ & $100 \%$ (antagonist $^{\dagger}$ ) & 25 \\
\hline \multicolumn{4}{|l|}{ Non-NETs } \\
\hline \multirow[t]{2}{*}{ Prostate cancer } & GRP & $>90 \%$ & 16,17 \\
\hline & PSMA* $^{*}$ & $>90 \%$ & 18 \\
\hline \multirow[t]{3}{*}{ Breast cancer } & GRP & $65 \%-75 \%$ & 20,23 \\
\hline & NPY (Y1 subtype) & $66 \%-85 \%$ & 22 \\
\hline & Somatostatin $\left(\mathrm{sst}_{2}\right)$ & $>90 \%$ (antagonist $^{\dagger}$ ) & $23-25$ \\
\hline \multirow[t]{3}{*}{ GIST } & GRP & $84 \%$ & 26 \\
\hline & CCK2 & $63 \%$ & 26 \\
\hline & VPAC2 & $84 \%$ & 26 \\
\hline \multirow[t]{2}{*}{ Ewing sarcoma } & Neurotensin (NT1 subtype) & $64 \%$ & 27 \\
\hline & NPY (Y1 subtype) & $84 \%$ & 28 \\
\hline \multirow[t]{2}{*}{ Glioblastoma } & Substance P (NK1) & $100 \%$ & 29 \\
\hline & NPY (Y2 subtype) & $83 \%$ & 30 \\
\hline $\begin{array}{l}\text { *Does not belong to } \\
{ }^{\dagger} \text { Tested with iodinat } \\
\text { VPAC2 = subtype } 2\end{array}$ & $\begin{array}{l}\text { ceptor family. } \\
\text { tatin receptor antagonist JR11. } \\
\text { ive intestinal polypeptide receptor; NK1 = }\end{array}$ & subtype of tachykini & \\
\hline
\end{tabular}




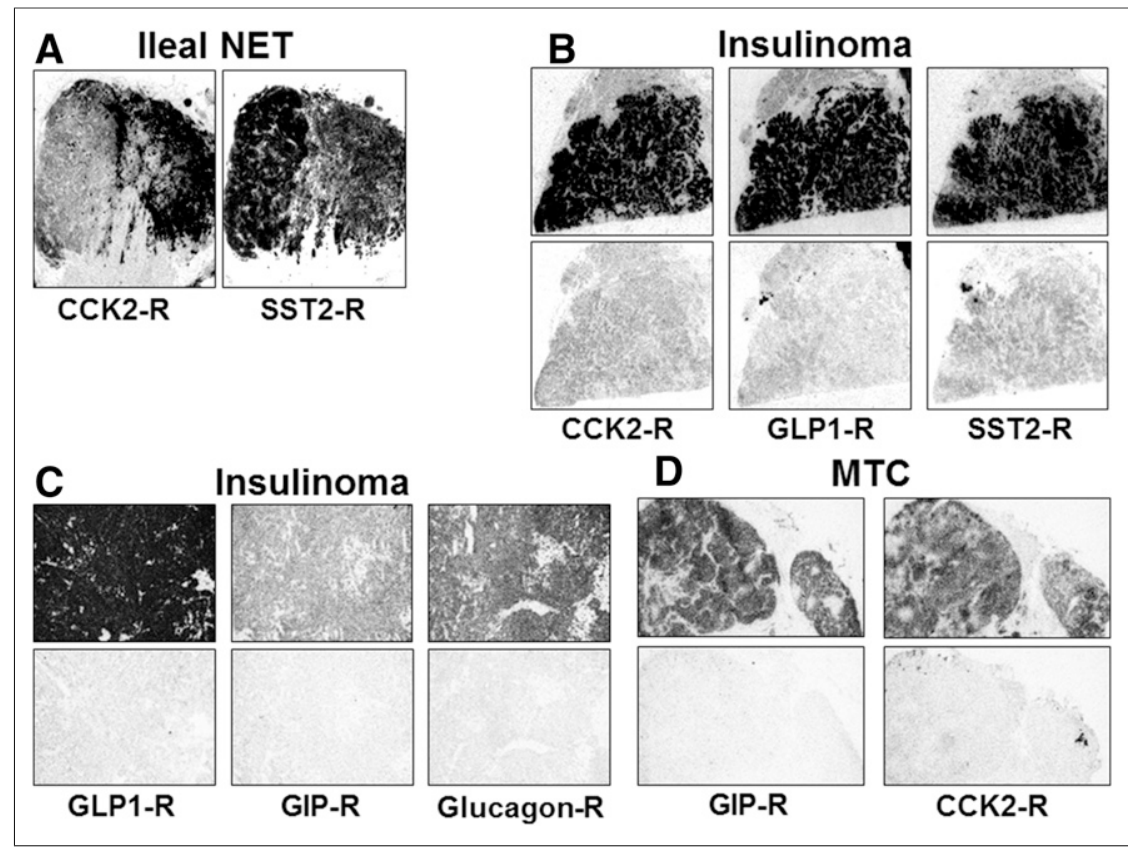

FIGURE 1. Expression of multiple receptors (R) in specific NETs.

GRP receptor imaging and PSMA imaging of prostate cancer can be successful (19).

\section{Breast Cancer}

Breast cancer is another example of cancer with multiple pepto be overexpressed in up to $75 \%$ of the breast cancer cases (20). Recent studies using radiolabeled GRP receptor antagonists suggest a high sensitivity of GRP receptor imaging in breast cancers (21). Equally abundant is the expression of the Y1 subtype of neuropeptide Y (NPY) receptors, overexpressed in $66 \%-85 \%$ of

\section{Ewing Sarcomas} tide receptor expression. Again, GRP receptors have been shown

cases $(22,23)$. Normal breast, including ducts and glands, also express NPY receptors, but fortunately of the Y2 subtype, suggesting that $\mathrm{Y} 1$ radioligands would specifically target the tumor (23). Figure 2A shows a breast tumor expressing GRP receptors as well as Y1. Also, somatostatin receptors have been shown previously to be present in breast cancers, however, in relatively low amounts $(23,24)$. A recently conducted study in vitro with an iodinated somatostatin receptor antagonist revealed, however, that a large amount of sst $_{2}$ could be detected in more than $90 \%$ of breast cancers (25).

The proof of concept in breast cancer patients has been shown with GRP receptors, NPY receptors, and somatostatin receptors. This large variety of overexpressed receptors suggests that various combinations of tracers may be suitable for imaging.

\section{GISTs}

GISTs have been shown in vitro with autoradiography to overexpress GRP receptors as well as CCK2 receptors (26). An example is shown in Figure 2B. These 2 target receptors were not investigated in vivo in GIST patients except for 1 study with GRP analogs that did not give the expected results. Also the VPAC2 subtype of the vasoactive intestinal polypeptide receptor is overexpressed in GISTs (Table 1). Because VPAC2 is much less often expressed in normal tissues than the VPAC1 subtype, the development of a VPAC2-selective radioligand may be meaningful.

The rare Ewing sarcomas, representing a huge challenge for treatment, are characterized by the overexpression of the NT1 subtype of neurotensin receptors in $64 \%$ of cases (27) as well as of the Y1 subtype of the NPY receptors in $84 \%$ of tumors (Table 1) (28). Figure 2C shows a representative example.

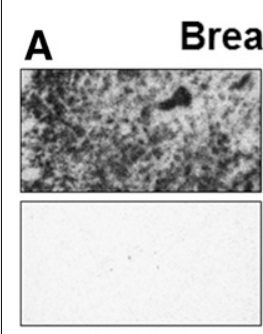

GRP-R

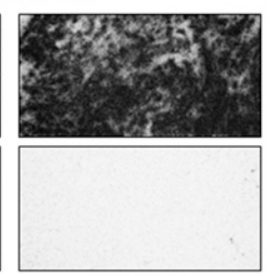

NPY-R

\section{Ewing sarcoma}

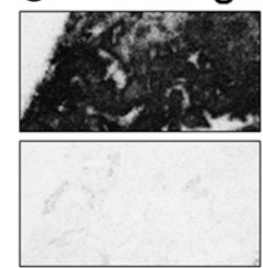

NPY-R
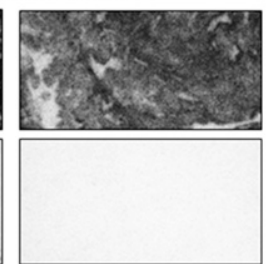

NT-R

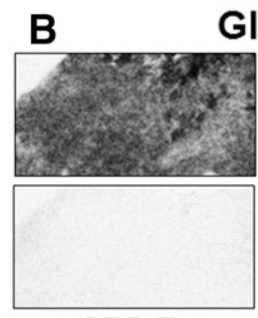

GRP-R
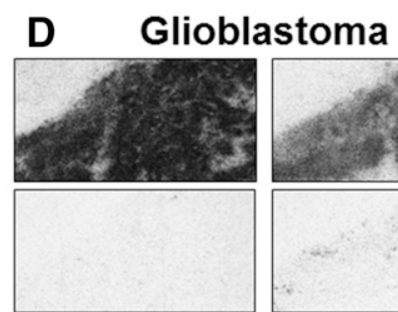

SP-R
FIGURE 2. Expression of multiple receptors $(R)$ in specific cancer types. GIST = gastrointestinal stromal tumors; NT = neurotensin; $\mathrm{SP}=$ substance $\mathrm{P} ; \mathrm{Tu}=$ tumors.

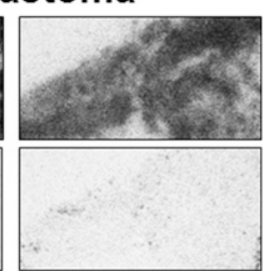

NPY-R

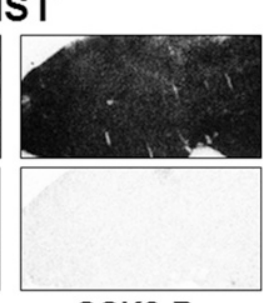

CCK2-R

\section{Glioblastomas}

At least 2 peptide receptors are overexpressed in glioblastomas in large amounts: substance $P$ receptors (also referred to as the neurokinin 1 subtype of tachykinin receptors (29)) and the Y1 subtype of NPY receptors (30). Figure 2D shows a case of glioblastoma coexpressing both receptors. Because of the blood-brain barrier, a successful targeting strategy with substance $\mathrm{P}$ analogs does not consist in a systemic application but in a local application into the tumor or in the cavity left by the resected tumor (31). Although substance $\mathrm{P}$ analogs were successfully applied in vivo in glioblastomas, NPY analogs have never been tested in glioblastomas.

\section{MULTIRECEPTOR TARGETING IN VIVO}

On the basis of the evidence that specific cancers express 2 or more peptide receptors 


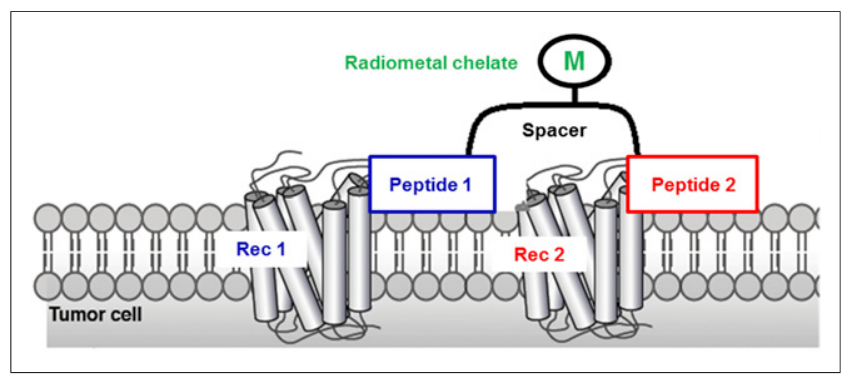

FIGURE 3. Schematic presentation of heterobivalent peptide connected via flexible spacer and simultaneously binding to 2 different $\mathrm{G}$ protein-coupled receptors (Rec). $\mathrm{M}=$ radiometal.

(Table 1), and that for many of these receptors the proof of concept that they can be individually imaged in vivo exists, it is attractive to think about the possibilities to target multiple receptors concomitantly in vivo in patients. There are 3 different approaches for multireceptor targeting in vivo: the use of heterobivalent or heteromultivalent ligands, which bind (simultaneously) to different receptors; the cocktail approach (coinjection of multiple tracers); and sequential injection of different imaging agents (enabling quantitative measurements of the pharmacokinetics of each tracer and allowing a decision about which tracer combination should be used for therapy [theranostic approach]).

\section{Heterobivalent or Heteromultivalent Ligands}

A heterobivalent or heteromultivalent ligand binds to 2 (multi) receptors through simultaneous, noncovalent interactions with 2 (multi) pharmacophores. For the sake of simplicity we focus mainly on heterobivalent ligands. This approach has the following 6 (theoretic) advantages relative to monovalent ligands.

The first advantage is improvement of the sensitivity of detection through a synergistic increase in binding affinities to targets. The higher affinity arises mainly from the simultaneous binding, which increases the bond enthalpies of 2 ligand receptor bonds, as well as slower dissociation from the tumor cell due to rebinding. This may be important for imaging at the later time points after background clearance. More importantly using heterobivalent ligands for therapeutic applications may result in higher tumor doses.

The second advantage is higher specificity/selectivity, as high affinity results only if the heterobivalent ligands bind simultaneously to their respective binding sites at the same cell. This is understood as binding of 1 pharmacophore forces the second one covalently attached via the linker to stay close to its binding molecule. This is called forced proximity, which favors not only binding but also rebinding. Thorough discussions on these aspects were published by Vauquelin et al. (32) and by Caplan et al. (33). Even if the spacer length or other structural shortcomings do not allow simultaneous binding to the 2 receptors a benefit may still arise due to the fact that a higher tumor uptake may result from the potential binding to a higher number of receptors available (receptor 1 and receptor 2), which allow monovalent binding of ligand 1 and ligand 2 . In addition, there is an increased probability of rebinding when ligand 1 dissociates from its receptor: ligand 2 can bind to receptor 2 and vice versa.

Third, tumors are most often heterogeneous. The use of heteromultivalent ligands may allow a more homogeneous distribution of radioligands with major consequences for targeted radionuclide therapy. An example of relative heterogeneity is demonstrated in Figure 1A.

Fourth, because of the larger size of heterodivalent constructs, the metabolic stability of peptides as radioligands may be improved, leading to improved bioavailability.

Fifth, a single agent can be used to target multiple surface markers that may be differentially expressed on cancer cells during the progression of disease.

Sixth, the single-agent perspective may be advantageous with regard to regulatory issues because only a single molecule needs to be validated and approved.

\section{Development of Heterobivalent Ligands for Simultaneous Cross-Linking of 2 Receptors}

The highest gain in affinity and selectivity of heterobivalent ligands arises if both pharmacophores bind simultaneously to their respective binding sites. Therefore, the design and development of these constructs are most desirable. Schematically this approach is illustrated in Figure 3.

The most sophisticated and elegant approach was devised by Vagner et al. (34), Josan et al. (35), and $\mathrm{Xu}$ et al. (36). They performed a modeling study to determine the optimal linker length and calculated an optimal length of 2-5 nm for 2 well-packed G-protein-coupled receptors and concluded that linker length and flexibility are the most important parameters. Vagner et al. (34) studied different pharmacophores targeting the $\delta$-opioid receptors and the human melanocortin- 4 receptor and found that affinity enhancement may be as high as 48 -fold if optimized linkers are used. In a melanocortin-1 receptor and CCK2 mouse model, a linker length of about $5 \mathrm{~nm}$ showed optimal tumor uptake and retention and in vitro a 12-fold-higher specificity for dualcompared with single-target receptor $(35,36)$.

Kroll et al. (37) have used a somewhat different approach to targeting GRP receptors. They developed hybrid bombesin analogs combining an agonist and an antagonist (Fig. 4). Their modular approach was based on a rigid oligoproline scaffold with defined distances. They aimed at combining the best of the agonist (internalization) and antagonist (recognition of more binding sites than agonists) world of GPCRs. Schematically this approach is illustrated in Figure 4. The heterobivalent ligand shows distinctly higher cell uptake in vitro than the homobivalent compounds. In vivo high and long-lasting tumor uptake resulted if the approximate peptide distance is around $2 \mathrm{~nm}$, well correlating with the calculations of Caplan et al. (33).

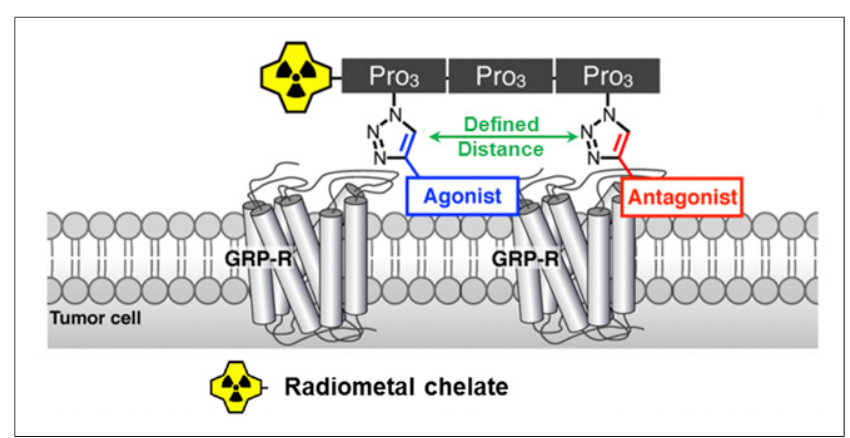

FIGURE 4. General concept of oligoproline-based hybrid ligands that allowed combination of homo- and heterovalent ligands at defined distances. Oligoprolines served as rigid scaffolds that allowed tailoring of distances between pharmacophores. $\mathrm{R}=$ receptors. 


\section{Development of Heterobivalent Ligands with Short Linkers}

Simultaneously binding ligands are difficult to design because of the choice of the ideal linker length, its rigidity and flexibility, respectively, allowing simultaneous binding with only small entropy loss. Therefore, several groups relied on the synthesis of constructs with short linkers, such as glutamic acid, $\beta$-aminoglutaric acid, or lysine connecting 2 different pharmacophores. This approach relies not on simultaneous binding but on a statistical advantage of these constructs to bind to a larger number of receptors. In addition, this approach benefits from a contribution via rebinding, which increases the tumor retention time.

This approach was chosen by different groups (38-40). The preferred "coligand" was the $\alpha_{V} \beta_{3}$-targeting RGD motif. The integrin family is expressed on tumor neovasculature but sometimes moderate expression on some human tumor cells is found. One of these constructs made its way into the clinic.

RGD-based peptides have been coupled via a Glu spacer to the GRP receptor-targeting peptide bombesin(7-14). The glutamic acid spacer allows also coupling of a chelator such as DOTA or NOTA for radiometal labeling or a prosthetic group for ${ }^{18} \mathrm{~F}$ labeling. These constructs were carefully tested in vitro and in animal models. One of these hybrids labeled with ${ }^{68} \mathrm{Ga}$ was translated to the clinic and compared with ${ }^{68} \mathrm{Ga}$-bombesin(7-14) in 13 prostate cancer patients and 5 volunteers (41). ${ }^{68} \mathrm{Ga}-\mathrm{BBN}-\mathrm{RGD}$ detected distinctly more metastatic lesions. This result is very promising and may even be improved if a GRP receptor antagonist is used. Stott Reynolds et al. used a similar construct with the GRP receptor antagonist RM2 (42). This conjugate shows very favorable pharmacokinetics.

PSMA is currently the most promising target for prostate cancer imaging. Shallal et al. combined a urea-based small molecule with an $\alpha_{\mathrm{v}} \beta_{3}$-targeted peptide and DOTA for radiolabeling. Preliminary affinity data and in vivo data with a conjugated dye show the high potential of these constructs for further development (40). Potentially the most promising approach in prostate cancer imaging is the combination of agents targeting PSMA or GRP receptors, as elegantly shown by Liolios et al. (43).

Apart from the described examples there have been many more heterobivalent ligands discussed in the 2 review articles $(44,45)$

\section{Current and Future Developments}

A fascinating current development that may be called "a combination boom" (46) is happening in the diabetes/obesity research field. It may potentially be exploited directly by medicinal radiochemists to develop new heteromultivalent radioligands based on peptides. Finan et al. developed monomeric triagonist peptides targeting the glucagon, GLP-1, and GIP receptors (47). They combined 3 peptides into a single molecule, circumventing the use of different peptidic drugs. This approach is shown in Figure 5. Figure $1 \mathrm{C}$ shows a tumor expressing precisely these 3 receptors.

They also studied dual agonists (GIP/GLP-1) formulated into a single peptide (48). With the use of (radio)chemical strategies developed before for agents targeting GLP-1 $(9,49)$ and GIP (11) receptors, a series of radioligands may be developed as imaging (and possibly radiotherapeutic) agents targeting tumors expressing these receptors simultaneously (11). Receptor families with different receptor subtypes such as the somatostatin receptors may also benefit from a similar approach. Radiolabeled multireceptor or even "pan" ligands, which target several (all) subtypes, were developed. They may lead to a higher diagnostic sensitivity than a single receptor-targeting agent, as demonstrated by Wild et al. (6)

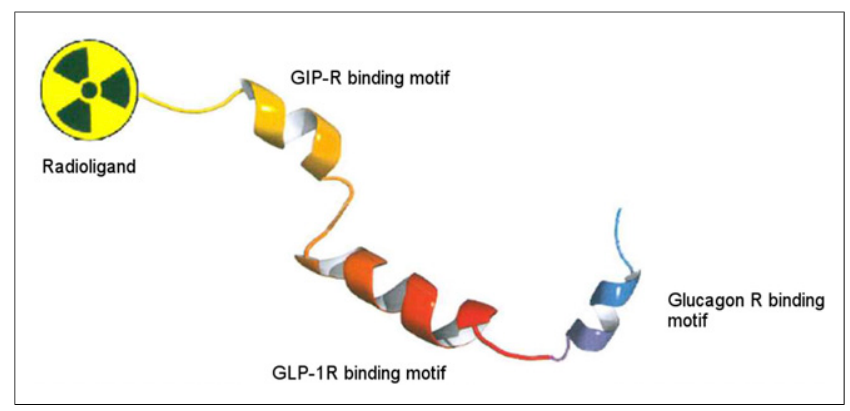

FIGURE 5. Schematic illustration of monomeric peptide triagonist targeting 3 different receptors $(\mathrm{R})$.

with the sst $_{2,3,5}$-targeting PET agent ${ }^{68} \mathrm{Ga}$-DOTANOC. The approaches outlined here may be implemented in future developments of ligands targeting peptide receptors. For instance, as reported by Reubi et al., GLP-1 receptors, GIP receptors, and $\mathrm{sst}_{2}$ may be concomitantly expressed in GEP NETs (14).

\section{Cocktail Approach}

The cocktail/combination strategy is regularly being used in classical cytotoxic chemotherapy. It is characterized by coadministration of combinations of multiple therapeutic agents (50).

The rationale of combination approaches is to achieve additive or even synergistic effects while minimizing toxicity. This may be achieved using drugs acting via different mechanisms in the tumor and in off-target organs (51). The same also holds for targeted radionuclide therapy.

The cocktail approach lacks some of the aforementioned advantages, such as rebinding and increased avidity due to simultaneous binding as well as the increased probability of using a heterobivalent short linker construct.

In the imaging field, cocktails of imaging agents are not frequently used. Their potential advantages are an increased sensitivity and simultaneous localization of disease with only 1 injection. There are only a few examples of the use of the cocktail approach in the clinic, such as using SPECT agents for the localization of parathyroid adenomas and medullary thyroid cancer as well as some cardiac nuclear imaging studies (52). In addition the Stanford group advocated for the combined administration of ${ }^{18} \mathrm{~F}-\mathrm{NaF}$ and ${ }^{18} \mathrm{~F}-\mathrm{FDG}$ in a single PET scan and initiated a prospective international multicenter trial (53). The authors concluded that the cocktail shows high sensitivity, lower cost, increased patient comfort, and reduced radiation exposure when compared with separate scans. Otherwise the modality is underused and almost excluded in PET for obvious reasons. Optical imaging has a big potential within the cocktail approach as simultaneous multicolor imaging can be performed and used for localization and characterization of expression profiles (52).

\section{Modifications of Cocktail Approach: Sequential Application of Different Imaging Agents}

The approach often used in nuclear medicine is a sequential application of different imaging agents usually with the aim to compare different radiopharmaceuticals in regard to their sensitivity and specificity. An impressive example showing the application of 6 tracers in a single patient with recurrent prostate cancer was presented at the 2016 SNMMI annual meeting in the highlights lecture (Fig. 6) (54). 


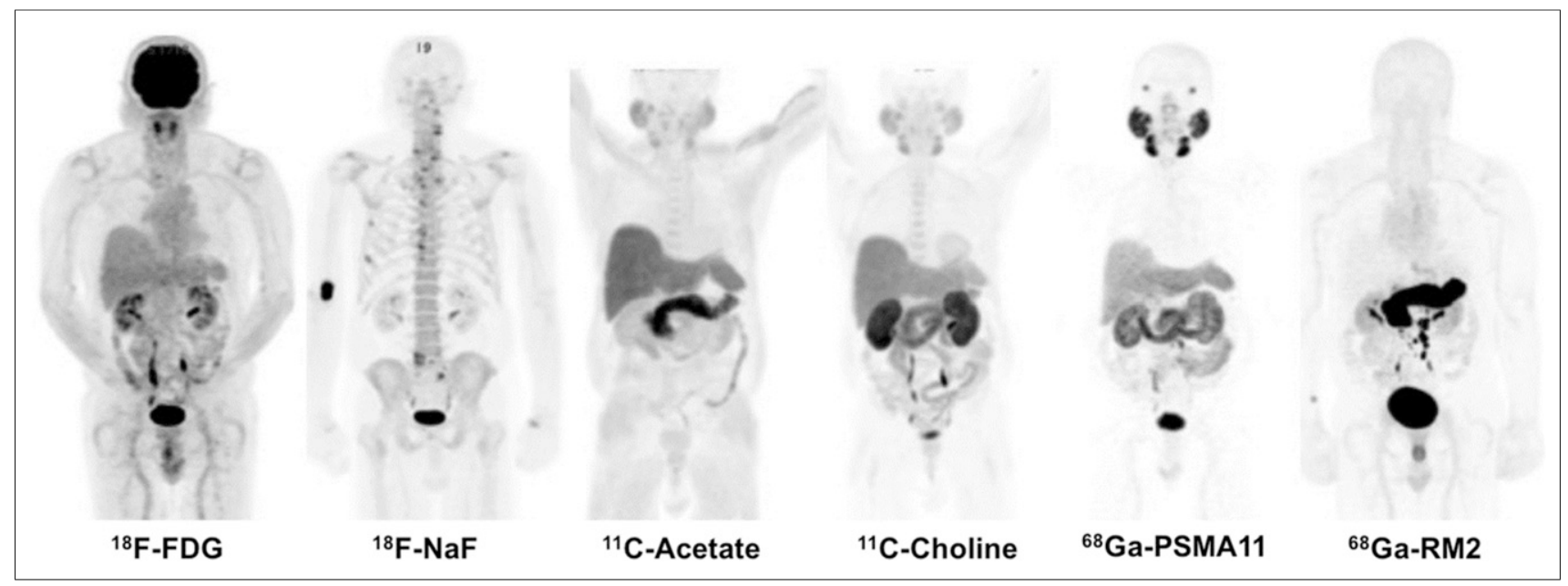

FIGURE 6. Images of 83-y-old man with biochemically recurrent prostate cancer (based on increase in prostate-specific antigen level). Images represent impressive examples of PET tracers injected in same patient at different time points (53). (Courtesy of Andrei lagaru, MD, Stanford University.)

Of particular interest are the $2{ }^{68} \mathrm{Ga}$-labeled PET tracers targeting PSMA and GRP receptors. The tracers target different biologic processes and therefore do not have the same sensitivity with regard to lymph node detection. It appears that GRP receptors are targets highly expressed already at an early stage of tumor development (17), whereas PSMA is highly expressed in patients with high Gleason scores. Therefore, patients may benefit from both tracers, and the development and optimization of heterobivalent compounds seem to be warranted, as does the use of a cocktail if a single-agent image is not conclusive.

\section{CONCLUSION}

Many human tumors overexpress different GPCRs, sometimes at high density. To target them simultaneously would increase diagnostic sensitivity and specificity. In addition targeting multiple receptors may alleviate some of the major problems in cancer targeting: heterogeneity, resistance, and change of phenotype during disease progression. The development and clinical translation of heterobivalent or multivalent ligands is a very promising approach although the design of simultaneously binding ligands may be challenging as simultaneous binding depends on many different factors such as receptor density, distance, and in particular the composition of the linker connecting the 2 pharmacophores. Still the approach is promising and simultaneous binding is not a "conditio sine qua non." Several of the most promising preclinically tested ligands should be translated to the clinic.

\section{DISCLOSURE}

No potential conflict of interest relevant to this article was reported.

\section{ACKNOWLEDGMENTS}

We thank Svetlana Rylova, $\mathrm{PhD}$, and Mireille Kuonen for support in the careful editing of the article.

\section{REFERENCES}

1. Ito T, Jensen RT. Molecular imaging in neuroendocrine tumors: recent advances, controversies, unresolved issues, and roles in management. Curr Opin Endocrinol Diabetes Obes. 2017;24:15-24.
2. Reubi JC. Peptide receptors as molecular targets for cancer diagnosis and therapy. Endocr Rev. 2003;24:389-427.

3. Ginj M, Zhang H, Waser B, et al. Radiolabeled somatostatin receptor antagonists are preferable to agonists for in vivo peptide receptor targeting of tumors. Proc Natl Acad Sci USA. 2006;103:16436-16441.

4. Reubi JC, Waser B, Schaer JC, Laissue JA. Somatostatin receptor sst1-sst5 expression in normal and neoplastic human tissues using receptor autoradiography with subtype-selective ligands. Eur J Nucl Med. 2001;28:836-846.

5. Wild D, Schmitt JS, Ginj M, et al. DOTA-NOC, a high-affinity ligand of somatostatin receptor subtypes 2, 3 and 5 for labelling with various radiometals. Eur J Nucl Med Mol Imaging. 2003;30:1338-1347.

6. Wild D, Bomanji JB, Benkert P, et al. Comparison of ${ }^{68} \mathrm{Ga}$-DOTANOC and ${ }^{68} \mathrm{Ga}-$ DOTATATE PET/CT within patients with gastroenteropancreatic neuroendocrine tumors. J Nucl Med. 2013;54:364-372.

7. Reubi JC, Waser B. Concomitant expression of several peptide receptors in neuroendocrine tumors as molecular basis for in vivo multireceptor tumor targeting. Eur J Nucl Med Mol Imaging. 2003;30:781-793.

8. Körner M, Stockli M, Waser B, Reubi JC. GLP-1 receptor expression in human tumors and human normal tissues: potential for in vivo targeting. $\mathrm{J} \mathrm{Nucl} \mathrm{Med.}$ 2007;48:736-743.

9. Wild D, Mäcke H, Christ E, Gloor B, Reubi JC. Glucagon-like peptide 1-receptor scans to localize occult insulinomas. N Engl J Med. 2008;359:766-768.

10. Waser B, Rehmann R, Sanchez C, Fourmy D, Reubi JC. Glucose-dependent insulinotropic polypeptide receptors in most gastroenteropancreatic and bronchial neuroendocrine tumors. J Clin Endocrinol Metab. 2012;97:482488.

11. Gourni E, Waser B, Clerc P, Fourmy D, Reubi JC, Maecke HR. The glucosedependent insulinotropic polypeptide receptor: a novel target for neuroendocrine tumor imaging-first preclinical studies. J Nucl Med. 2014;55:976-982.

12. Reubi JC, Schaer JC, Waser B. Cholecystokinin(CCK)-A and CCK-B/gastrin receptors in human tumors. Cancer Res. 1997;57:1377-1386.

13. Behr TM, Behe MP. Cholecystokinin-B/gastrin receptor-targeting peptides for staging and therapy of medullary thyroid cancer and other cholecystokinin-B receptor-expressing malignancies. Semin Nucl Med. 2002;32:97-109.

14. Reubi JC, Waser B. Triple-peptide receptor targeting in vitro allows detection of all tested gut and bronchial NETs. J Nucl Med. 2015;56:613-615.

15. Waser B, Beetschen K, Pellegata NS, Reubi JC. Incretin receptors in nonneoplastic and neoplastic thyroid $\mathrm{C}$ cells in rodents and humans: relevance for incretin-based diabetes therapy. Neuroendocrinology. 2011;94:291-301.

16. Markwalder R, Reubi JC. Gastrin-releasing peptide receptors in the human prostate: relation to neoplastic transformation. Cancer Res. 1999;59:1152-1159.

17. Körner M, Waser B, Rehmann R, Reubi JC. Early over-expression of GRP receptors in prostatic carcinogenesis. Prostate. 2014;74:217-224.

18. Sweat SD, Pacelli A, Murphy GP, Bostwick DG. Prostate-specific membrane antigen expression is greatest in prostate adenocarcinoma and lymph node metastases. Urology. 1998;52:637-640.

19. Minamimoto R, Hancock S, Schneider B, et al. Pilot comparison of ${ }^{68} \mathrm{Ga}-\mathrm{RM} 2$ PET and ${ }^{68}$ Ga-PSMA-11 PET in patients with biochemically recurrent prostate cancer. J Nucl Med. 2016;57:557-562. 
20. Gugger M, Reubi JC. GRP receptors in non-neoplastic and neoplastic human breast. Am J Pathol. 1999;155:2067-2076.

21. Stoykow C, Erbes T, Maecke HR, et al. Gastrin-releasing peptide receptor imaging in breast cancer using the receptor antagonist ${ }^{68} \mathrm{Ga}-\mathrm{RM} 2$ and PET. Theranostics. 2016;6:1641-1650.

22. Reubi JC, Gugger M, Waser B, Schaer JC. Y1-mediated effect of neuropeptide Y in cancer: breast carcinomas as targets. Cancer Res. 2001;61:4636-4641.

23. Reubi C, Gugger M, Waser B. Coexpressed peptide receptors in breast cancers as molecular basis for in vivo multireceptor tumor targeting. Eur J Nucl Med Mol Imaging. 2002;29:855-862.

24. Albérini JL, Meunier B, Denzler B, et al. Somatostatin receptor in breast cancer and axillary nodes: study with scintigraphy, histopathology and receptor autoradiography. Breast Cancer Res Treat. 2000;61:21-32.

25. Reubi JC, Waser B, Mäcke H, Rivier J. Highly increased ${ }^{125}$ I-JR11 antagonist binding in vitro reveals novel indications for sst2 targeting in human cancers. J Nucl Med. 2017;58:300-306.

26. Reubi JC, Korner M, Waser B, Mazzucchelli L, Guillou L. High expression of peptide receptors as a novel target in gastrointestinal stromal tumours. Eur J Nucl Med Mol Imaging. 2004;31:803-810.

27. Reubi JC, Waser B, Schaer JC, Laissue JA. Neurotensin receptors in human neoplasms: high incidence in Ewing sarcomas. Int J Cancer. 1999;82:213-218.

28. Körner M, Waser B, Reubi JC. High expression of neuropeptide y1 receptors in Ewing sarcoma tumors. Clin Cancer Res. 2008;14:5043-5049.

29. Hennig IM, Laissue JA, Horisberger U, Reubi JC. Substance P receptors in human primary neoplasms: tumoural and vascular localisation. Int $J$ Cancer. 1995;61:786-792.

30. Körner M, Reubi JC. Neuropeptide Y receptors in primary human brain tumors: overexpression in high-grade tumors. J Neuropathol Exp Neurol. 2008;67:741-749.

31. Kneifel S, Cordier D, Good S, et al. Local targeting of malignant gliomas by the diffusible peptidic vector 1,4,7,10-tetraazacyclododecane-1-glutaric acid-4,7,10triacetic acid-substance P. Clin Cancer Res. 2006;12:3843-3850.

32. Vauquelin G, Charlton SJ. Exploring avidity: understanding the potential gains in functional affinity and target residence time of bivalent and heterobivalent ligands. Br J Pharmacol. 2013;168:1771-1785.

33. Caplan MR, Rosca EV. Targeting drugs to combinations of receptors: a modeling analysis of potential specificity. Ann Biomed Eng. 2005;33:1113-1124.

34. Vagner J, Xu L, Handl HL, et al. Heterobivalent ligands crosslink multiple cellsurface receptors: the human melanocortin-4 and delta-opioid receptors. Angew Chem Int Ed Engl. 2008;47:1685-1688.

35. Josan JS, Handl HL, Sankaranarayanan R, et al. Cell-specific targeting by heterobivalent ligands. Bioconjug Chem. 2011;22:1270-1278.

36. Xu L, Josan JS, Vagner J, et al. Heterobivalent ligands target cell-surface receptor combinations in vivo. Proc Natl Acad Sci USA. 2012;109:21295-21300.

37. Kroll C, Mansi R, Braun F, Dobitz S, Maecke HR, Wennemers H. Hybrid bombesin analogues: combining an agonist and an antagonist in defined distances for optimized tumor targeting. J Am Chem Soc. 2013;135:16793-16796.
38. Li ZB, Wu Z, Chen K, Ryu EK, Chen X. ${ }^{18} \mathrm{~F}-$ labeled BBN-RGD heterodimer for prostate cancer imaging. J Nucl Med. 2008;49:453-461.

39. Liu Z, Yan Y, Liu S, Wang F, Chen $\mathrm{X} .{ }^{18} \mathrm{~F},{ }^{64} \mathrm{Cu}$, and ${ }^{68} \mathrm{Ga}$ labeled RGD-bombesin heterodimeric peptides for PET imaging of breast cancer. Bioconjug Chem. 2009; 20:1016-1025.

40. Shallal HM, Minn I, Banerjee SR, Lisok A, Mease RC, Pomper MG. Heterobivalent agents targeting PSMA and integrin- $\alpha_{\mathrm{v}} \beta_{3}$. Bioconjug Chem. 2014;25: 393-405.

41. Zhang J, Niu G, Lang L, et al. Clinical translation of a dual integrin $\alpha_{\mathrm{v}} \beta_{3}$ - and gastrin-releasing peptide receptor-targeting PET radiotracer, ${ }^{68} \mathrm{Ga}-\mathrm{BBN}-\mathrm{RGD}$. J Nucl Med. 2017;58:228-234.

42. Stott Reynolds TJ, Schehr R, Liu D, et al. Characterization and evaluation of DOTA-conjugated bombesin/RGD-antagonists for prostate cancer imaging and therapy. Nucl Med Biol. 2015;42:99-108.

43. Liolios C, Schäfer M, Haberkorn U, et al. Novel bispecific PSMA/GRPr targeting radioligands with optimized pharmacokinetics for improved PET imaging of prostate cancer. Bioconjug Chem. 2016;27:737-751.

44. Fischer G, Schirrmacher R, Wangler B, Wangler C. Radiolabeled heterobivalent peptidic ligands: an approach with high future potential for in vivo imaging and therapy of malignant diseases. ChemMedChem. 2013;8:883-890.

45. Yan Y, Chen X. Peptide heterodimers for molecular imaging. Amino Acids. 2011;41:1081-1092.

46. Chakradhar S. All in one: researchers create combination drugs for diabetes and obesity. Nat Med. 2016;22:694-696.

47. Finan B, Yang B, Ottaway N, et al. A rationally designed monomeric peptide triagonist corrects obesity and diabetes in rodents. Nat Med. 2015;21:27-36.

48. Finan B, Ma T, Ottaway N, et al. Unimolecular dual incretins maximize metabolic benefits in rodents, monkeys, and humans. Sci Transl Med. 2013;5: 209ra151.

49. Rylova SN, Waser B, Del Pozzo L, et al. Approaches to improve the pharmacokinetics of radiolabeled glucagon-like peptide-1 receptor ligands using antagonistic tracers. J Nucl Med. 2016;57:1282-1288.

50. DeVita VT Jr, Young RC, Canellos GP. Combination versus single agent chemotherapy: a review of the basis for selection of drug treatment of cancer. Cancer. 1975;35:98-110.

51. Al-Lazikani B, Banerji U, Workman P. Combinatorial drug therapy for cancer in the post-genomic era. Nat Biotechnol. 2012;30:679-692.

52. Kobayashi H, Longmire MR, Ogawa M, Choyke PL, Kawamoto S. Multiplexed imaging in cancer diagnosis: applications and future advances. Lancet Oncol. 2010;11:589-595.

53. Iagaru A, Mittra E, Mosci C, et al. Combined ${ }^{18} \mathrm{~F}$-fluoride and ${ }^{18} \mathrm{~F}$-FDG PET/CT scanning for evaluation of malignancy: results of an international multicenter trial. J Nucl Med. 2013;54:176-183.

54. Weber WA. 2016 SNMMI Highlights Lecture: oncology, part 2. J Nucl Med. 2017;58:9N-15N. 